\title{
Implant Surgery for Fixed Implant-supported Prostheses in the Edentulous Mandible: A Case Report
}

\author{
Youngdeok Chee, DDS, MSD, PhD* \\ Professor, Department of Oral \& Maxillofacial Surgery, College of Dentistry, Wonkwang University, Iksan, Korea \\ *Corresponding author: Youngdeok Chee, Department of Oral \& Maxillofacial Surgery, Sanbone dental \\ hospital, College of Dentistry, Wonkwang University, 1142, Sanbon-dong, Gunpo-city, Gyeonggi-do \\ 15865 , Korea. \\ Tel:+82-31-390-2875. Fax : +82-31-390-2777. E-mail : omschee@wku.ac.kr
}

\section{OPEN ACCESS}

pISSN 2765-7833

eISSN 2765-7841

Journal of implantology and applied sciences

2021; 25(2): 84-94

https://doi.org/10.32542/implantology.2021009

Received: March 10, 2021

Accepted: March 30, 2021

ORCID

Youngdeok Chee

https://orcid.org/0000-0002-0333-4685

Copyright $\odot$ 2021. The Korean Academy of Oral \& Maxillofacial Implantology

This is an Open Access article distributed under the terms of the Creative Commons Attribution Non-Commercial License (http://creativecommons. org/licenses/by-nc/4.0/) which permits unrestricted non-commercial use, distribution, and reproduction in any medium, provided the original work is properly cited.

\begin{abstract}
Edentulism following extraction of existing teeth due to dental caries, severe periodontal disease, or surgical damage can be treated with implants using complete dentures, implant-supported overdentures, or fixed implant-supported prostheses. Fixed implant-supported prostheses have higher masticatory efficiency than removable prostheses and may provide patients with psychological stability. Moreover, when compared with traditional removable complete dentures, fixed implantsupported prostheses have better stability and retention, help improve the mental health of the patients, and provide them with opportunities to respond more actively to the society. In this report, patients preferred fixed implant-supported prostheses, which were advantageous in terms of masticatory efficiency, patient satisfaction, and distribution of stress compared to removable prostheses. Thus, they were considered the first priority. In case 1, a fixed implant-supported prosthesis was fabricated by placing 12 implants in a patient with edentulous mandible who was using a mandibular complete denture, but complained of denture displacement and masticatory dysfunction. In case 2, a fixed implant-supported prosthesis was fabricated by placing eight implants in a patient with an edentulous mandible whose entire mandibular dentition had been extracted due to severe periodontitis. Satisfactory functional and esthetic outcomes were achieved with fabrication and placement of fixed implant-supported prostheses in these patients.
\end{abstract}

Keywords: Dental implant, Edentulous mandible, Fixed prostheses

\section{I . Introduction}

Edentulism following extraction of existing teeth due to dental caries, severe periodontal disease, or surgical damage can be treated with implants using one of following three methods: complete dentures, implant-supported overdentures, and fixed implant-supported prostheses.

Although complete dentures may represent a traditional prosthetic restoration method for edentulous patients, most of the complaints regarding complete dentures include pain 
and discomfort caused by dentures, lack of stability, and decreased masticatory ability, which often lead to psychological dissatisfaction.

Implant-supported removable overdentures as well as fixed implant-supported prostheses utilize implants and may enhance, maintain, and stabilize masticatory ability and preserve the alveolar bone. Hence, they can be good alternatives to traditional dentures. ${ }^{1}$

Treatment options using implants are divided largely into removable and fixed. The removable option involves fabricating implant-supported overdentures, while the fixed option involves restoring the edentulous jaw with a fixed implant-supported prosthesis. Patient preference, social and economic factors, esthetic factors such as facial support and lip support, and jaw relations should be considered while selecting the treatment option. ${ }^{2}$

A fixed implant-supported prosthesis has a higher masticatory efficiency than a removable prosthesis and may provide patients with psychological stability. In addition, when compared with traditional removable complete dentures, a fixed implant-supported prosthesis provides enhanced stability and retention of the prosthesis and helps improve the mental health of patients. Furthermore, it sometimes provides patients with opportunities to respond more positively to the society. ${ }^{3,4}$

In case of edentulism at a younger age, fixed prosthetic restoration using multiple implants may result in higher psychological and functional satisfaction compared to complete dentures. ${ }^{5,6}$ With popularization of implants as well as enhanced interest and knowledge level of dental patients, the demand for treatment using implants is increasing. Particularly, treatment of completely edentulous patients using a fixed implant-supported prosthesis is advantageous for the stability and maintenance of the prosthesis, increased support, and enhanced masticatory efficiency compared to traditional complete dentures.

We report two cases with successful outcomes, which involved restoration of an edentulous mandible using a fixed implant-supported prosthesis. The first patient had complaints of denture displacement and masticatory dysfunction due to long-term use of a mandibular complete denture, while the second patient underwent extraction of the entire mandibular dentition due to severe periodontitis. We placed 12 and 8 implants in these two patients, respectively. Subsequently, we fabricated and installed fixed implant-supported prostheses, which led to good functional and esthetic outcomes.

\section{II . Case Report}

\section{Case 1}

A 55-year-old completely edentulous man visited our hospital for consultation regarding implant restoration. He had undergone extraction of all his teeth a long time ago and had been using a complete 
denture. However, he complained of inconvenience while using the denture and expressed a desire for a fixed implant-supported prosthesis for the edentulous mandible. The panoramic radiograph showed that the mandibular bone was maintained sufficiently and a surgical plan was established to fabricate a fixed prosthesis following placement of 12 implants in the mandible without additional bone graft (Fig. 1). Local anesthesia (2\% lidocaine with 1:100,000 epinephrine; Yuhan, Seoul, Korea) was administered to the surgical site in the mandible. The incision sites for implant placement and periodontal flap reflection were determined by applying a surgical guide to ensure that all implants would be placed in the correct positions (Fig. 2A, 2B). A vertical incision was made at the middle and posterior of both the ends of the mandible, followed by a horizontal incision connecting the vertical incisions (Fig. 2C).

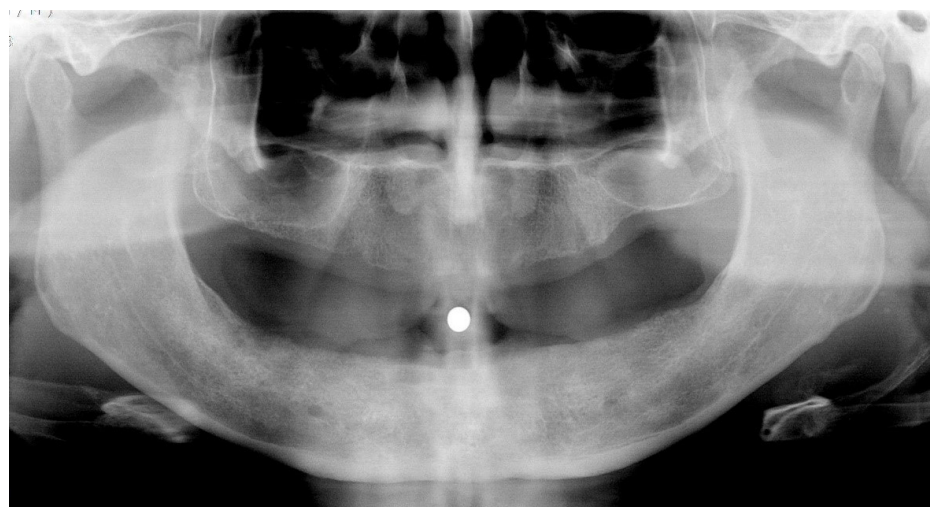

Fig. 1. Case l: Pre-operative panoramic radiograph.
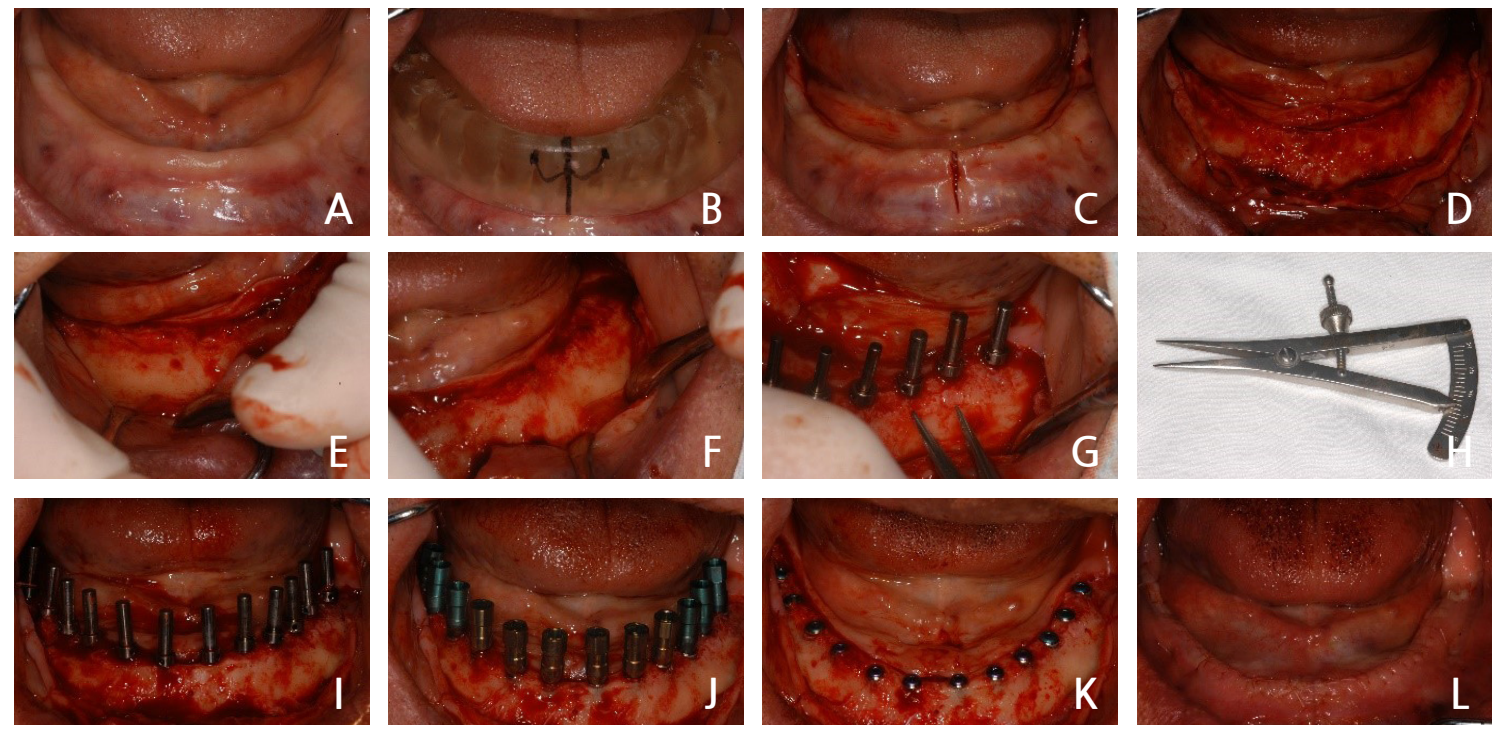

Fig. 2. Case I: Intraoral views of the first-stage implant surgery. (A) Pre-operative intraoral view, (B) surgical stent adaptation, (C) incision at the surgical site, (D) full-thickness flap elevation, (E) right mental foramen and nerve, (F) left mental foramen and nerve, (G) checking the inter-implant space using a castroviejo caliper, (H) castroviejo caliper, (I) guide pin insertion, (J) implant placement, (K) cover screw insertion after mount removal, (L) suturing the flap with continuous mattress and interrupted sutures. 
Flap reflection was performed and the periodontal flap was fixed to the mucous membrane using tagging sutures to ensure a good visibility of the surgical site even without retraction (Fig. 2D). After elevating the periodontal flap, the periosteum was carefully reflected to check the mental foramen and mental nerve underneath the premolars on both sides and their positions were confirmed to avoid nerve damage during surgery (Fig. 2E, 2F). Accurate drilling was performed at the positions of implant placement using a surgical stent. After $2 \mathrm{~mm}$ of drilling, a guide pin was inserted to confirm the position and direction of implant placement. Additionally, a castroviejo caliper was used to measure the inter-implant distance accurately and the implant placement position was determined (Fig. 2G, 2H, 2I). Subsequently, 6 implants with a diameter of $3.5 \mathrm{~mm}$ and a length of $11.5 \mathrm{~mm}\left(\mathrm{GS}-\mathrm{II}{ }^{\circledR}\right.$, Osstem, Seoul, Korea) were placed in the anterior region of the mandible and 3 implants with a diameter of $4.0 \mathrm{~mm}$ and a length of $10 \mathrm{~mm}\left(\mathrm{GS}-\mathrm{II}^{\circledR}\right.$, Osstem, Seoul, Korea) were placed in the molar region on both sides. Thus, 6 implants were placed in the molar region (Fig. 2J, 2K). After implant placement, the flap was sutured with primary closure using continuous mattress sutures and interrupted sutures (Fig. 2L). Healing proceeded well after the implant surgery. After 4 months, secondary implant surgery was performed to connect the healing abutment (Fig. 3A, 3B). After the secondary implant surgery, the fixed implant-supported prosthesis
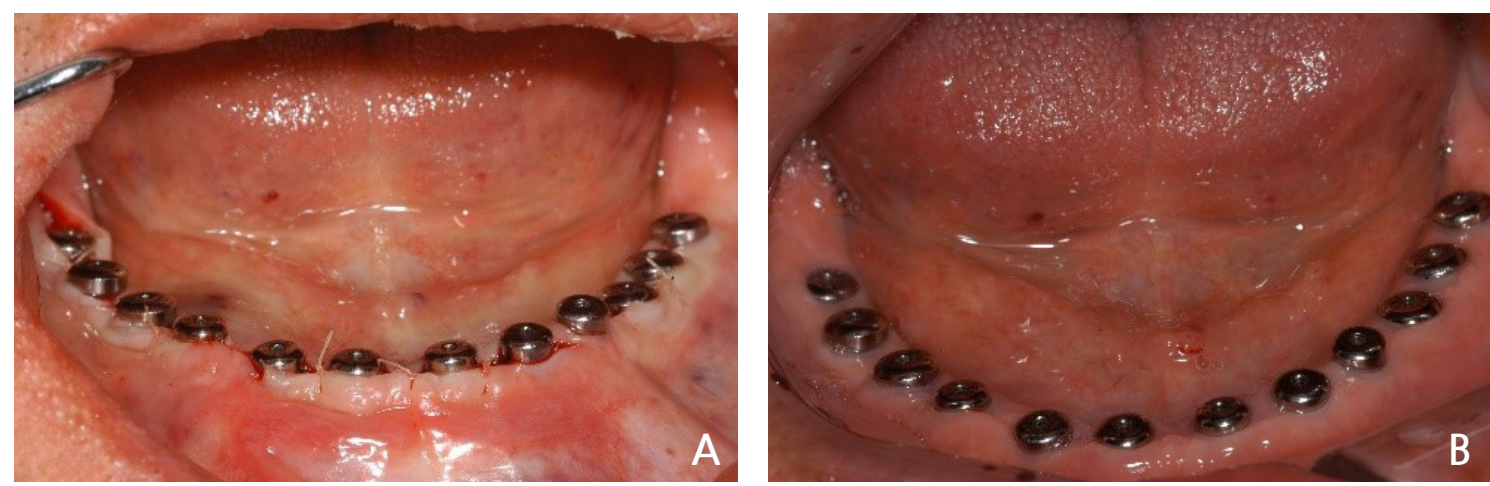

Fig. 3. Case I: Intraoral views of the second-stage implant surgery. (A) Interrupted sutures after the second-stage implant surgery, (B) healing stage after suture removal.

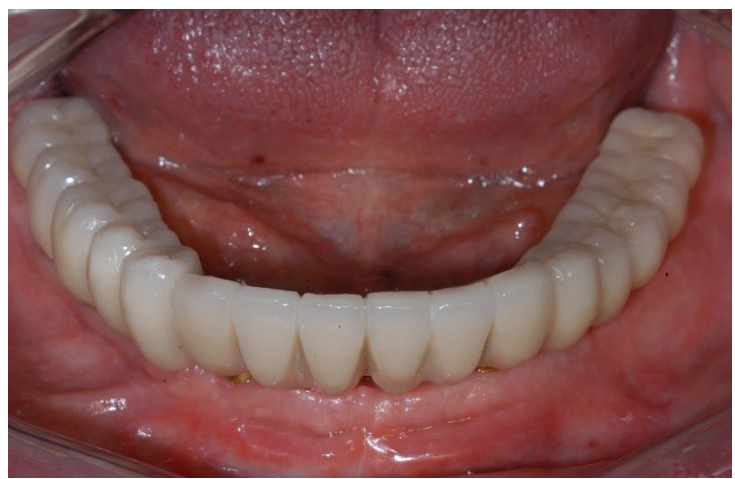

Fig. 4. Case l: Intraoral view of the final restoration. 
was fabricated and installed intraorally in the Department of Prosthodontics (Figs. 4 and 5). A panoramic radiograph obtained at 10 years after the prosthesis installation showed peri-implantitis and implant mobility with two implants in the left molar region and the patient complained of discomfort while mastication. Removal and replacement of the implants were planned (Fig. 6). Local anesthesia was administered to the surgical site (Fig. 7A) and the two implants with peri-implantitis were removed using mandibular forceps (Fig. 7B, 7C). Satisfactory wound healing was observed after removal of the

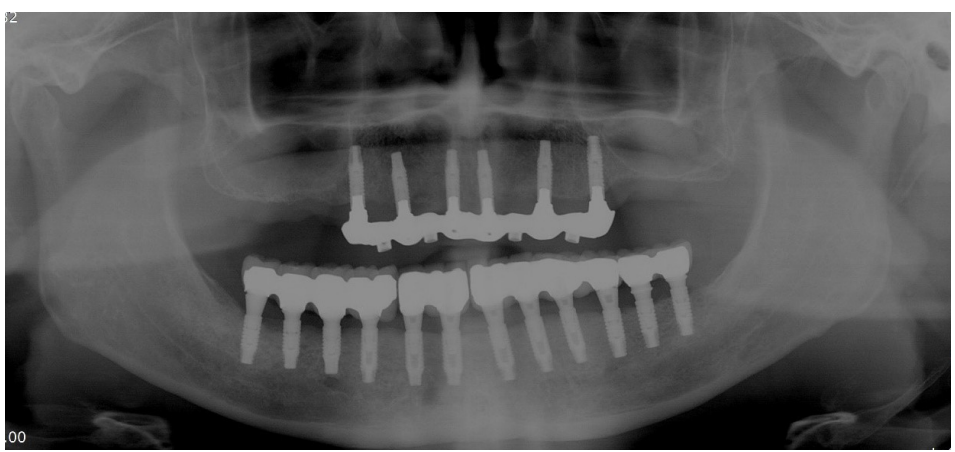

Fig. 5. Case l: Panoramic radiograph of the final restoration.
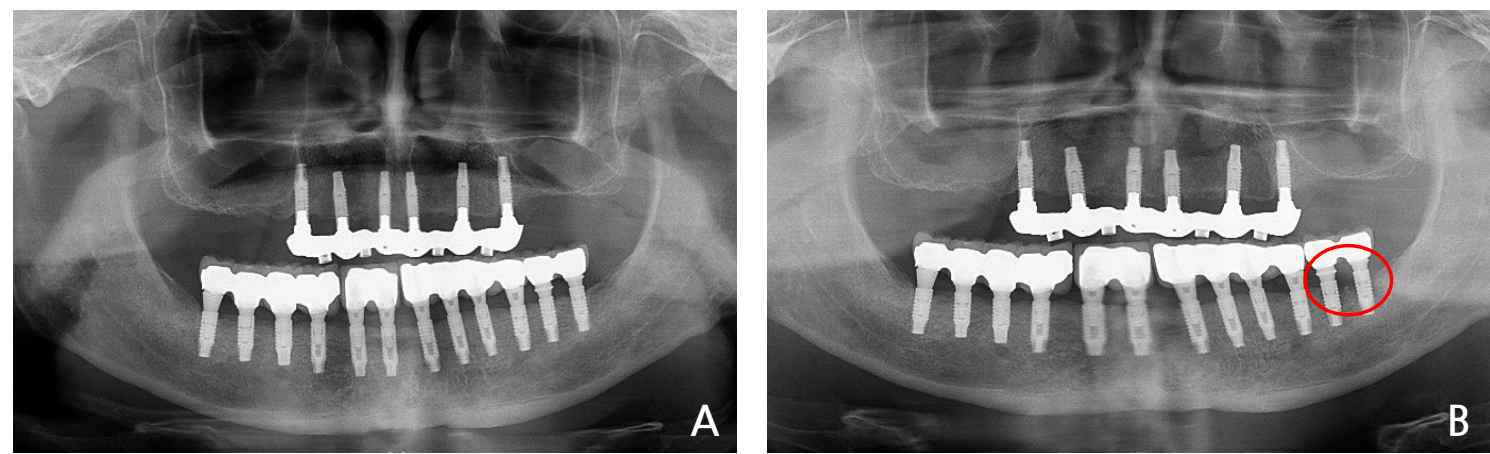

Fig. 6. Case l: Panoramic radiographs of the final restoration after 9 years (A) and after 10 years (B).
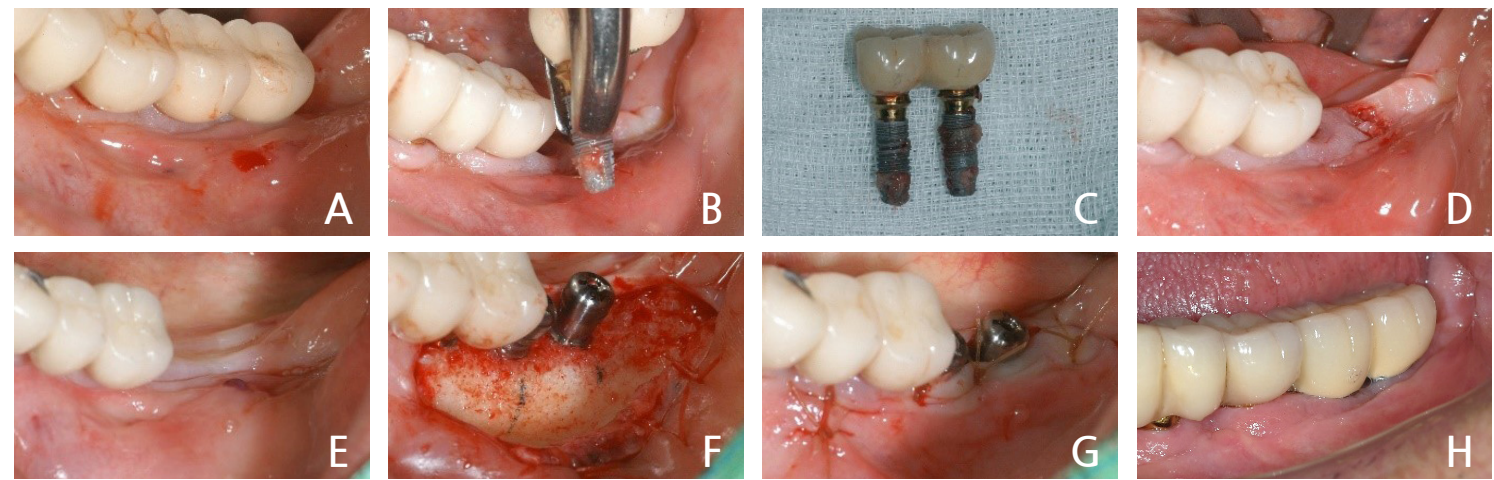

Fig. 7. Case I: Removal of the failed implants and implant replacement surgery. (A) Implants showing peri-implantitis, (B) implant removal, (C) removed implants, (D) intra-oral site after implant removal, (E) pre-operative site, $(F)$ implant replacement surgery with placement of healing abutment, $(G)$ suturing, $(\mathrm{H})$ final restoration. 
implants. After 3 months, implant replacement surgery was performed under local anesthesia (Fig. 7D, 7E). After elevation of the flap, two implants with a diameter of $4.5 \mathrm{~mm}$ and a length of $8 \mathrm{~mm}$ (Superline ${ }^{\circledR}$, Dentium, Seoul, Korea) were placed (Fig. 7F, 7G) and after 7 months, fixed implant-supported prosthesis was installed (Fig. 7H). The post-surgery panoramic radiograph showed correct placement of the implants and the post-prosthesis panoramic radiograph showed a good outcome (Fig. 8A, 8B). Followup observation at 15 years after the installation of the mandibular prosthesis revealed that the fixed implant-supported prosthesis was well maintained (Fig. 9). Panoramic radiographs also showed good results without bone loss (Fig. 10).
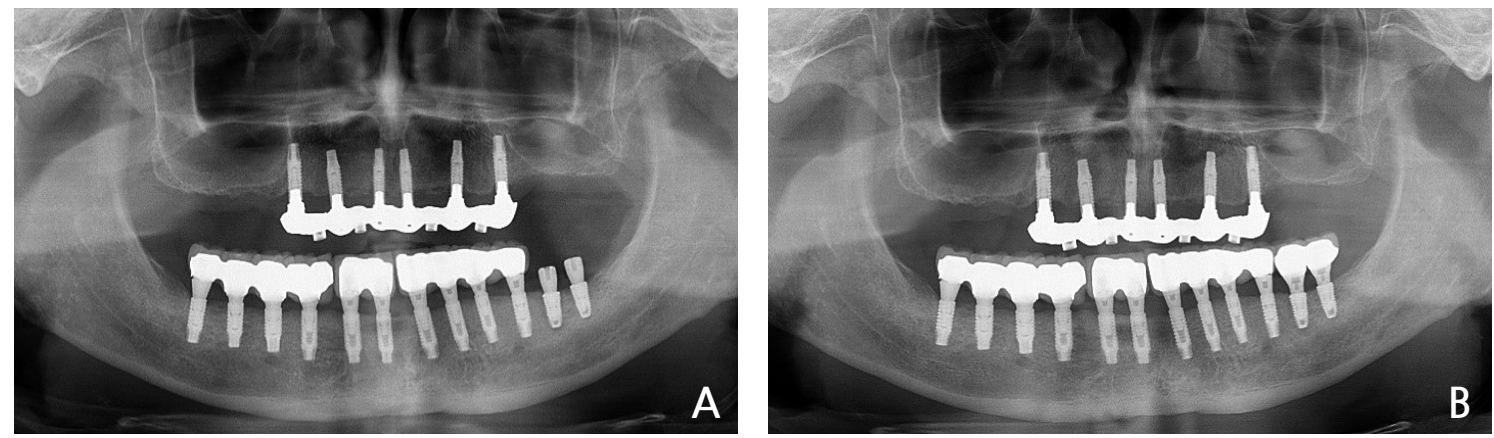

Fig. 8. Case I: Panoramic radiographs after the implant replacement surgery $(A)$ and after final restoration (B).

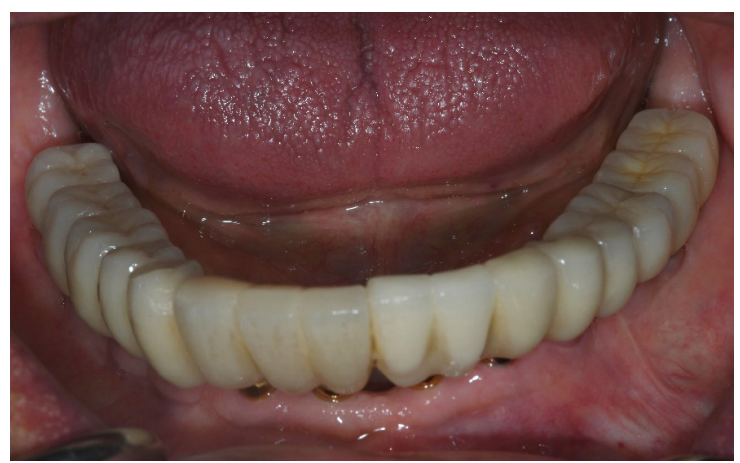

Fig. 9. Case l: Intraoral view of the final restoration after 15 years.

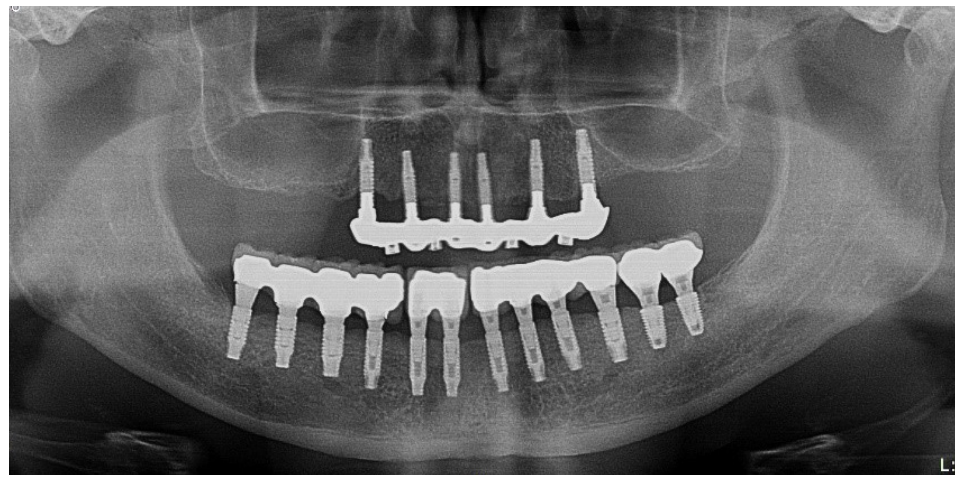

Fig. 10. Case l: Panoramic radiograph of the final restoration after 15 years. 


\section{Case 2}

A 45-year-old male patient with severe chronic periodontitis in the maxilla and mandible visited our hospital for consultation regarding implants. At his initial visit to this hospital, his oral condition was poor and he had difficulty in mastication due to residual tooth roots and severe periodontal disease. Consequently, placement of eight implants following extraction of the entire dentition and a subsequent fixed implant-supported prosthesis was planned (Fig. 11A, 11B). Maxillary and mandibular teeth were extracted under local anesthesia and the implant surgery was performed at 1 month after extractions (Fig. 12). After local anesthesia was administered to the surgical site in the mandible, flap elevation was performed by reflecting a full-thickness flap and periosteum to expose the mandible (Fig. 13A, 13B). After extraction, the uneven mandible was smoothed and the positions of the eight implants were determined. After drilling, a guide pin was inserted to confirm the direction and position of implant placement (Fig. 13C, 13D). Drilling was performed at the determined sites for implant placement. Two implants with a diameter of $3.8 \mathrm{~mm}$ and a length of $13 \mathrm{~mm}$ were placed in the anterior region, four implants with a diameter of $4.3 \mathrm{~mm}$ and a length of $13 \mathrm{~mm}$ were placed in the premolar region, and two implants with a diameter of $5.0 \mathrm{~mm}$ and a length of $13 \mathrm{~mm}$ were placed in the molar region (Camlog${ }^{\circledR}$, Camlog biotechnologies, Basel, Switzerland) (Fig. 13E). During the period of osseointegration, four
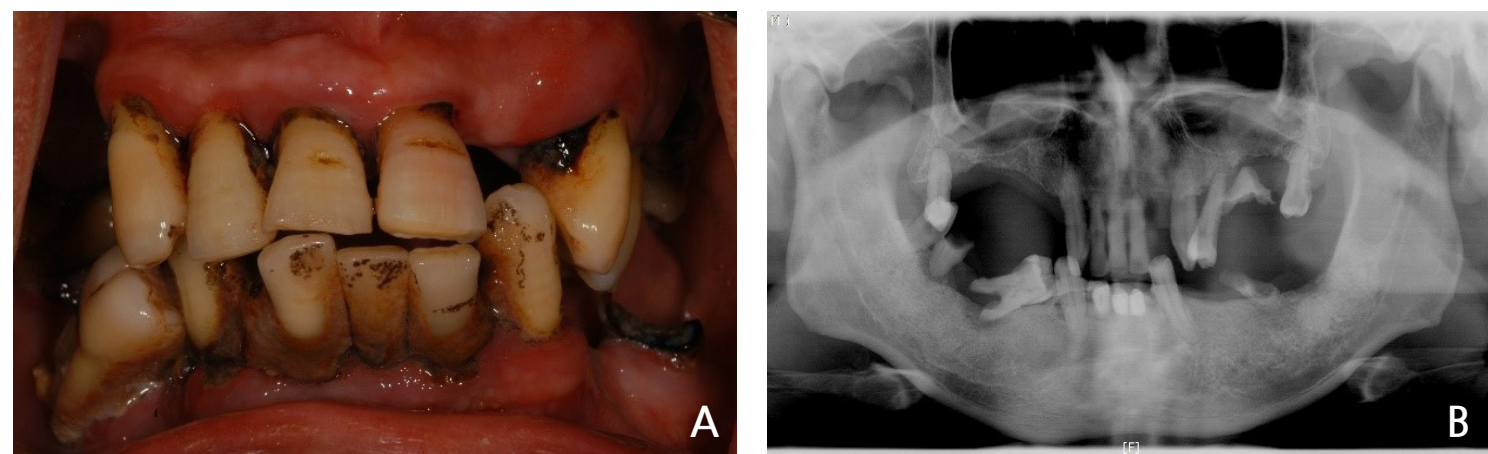

Fig. 11. Case II: Intraoral view (A) and panoramic view (B) at the first visit.

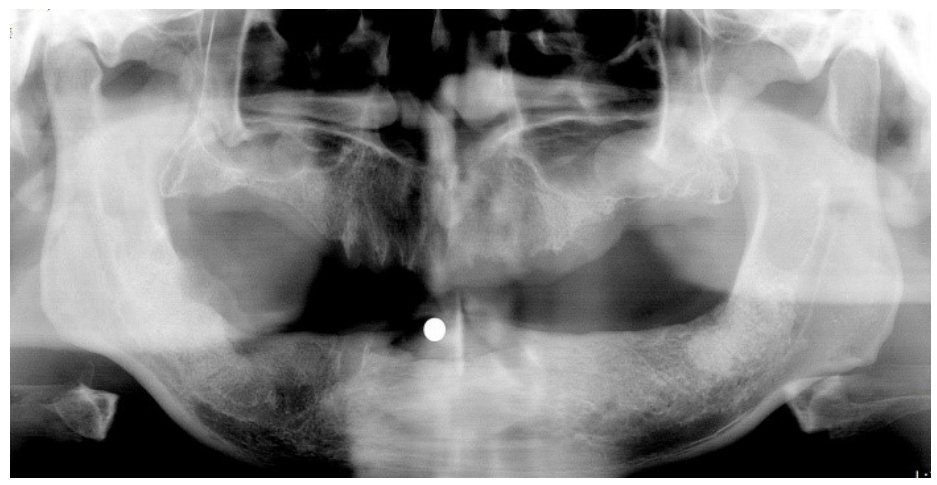

Fig. 12. Case Il: Pre-operative panoramic radiograph. 
temporary implants were placed to ensure that the temporary denture intended for use during this waiting period did not exert pressure on the implants and cause irritation (Fig. 13F). The surgical site was sutured with continuous mattress sutures and interrupted sutures. A temporary denture was fabricated and installed (Fig. 13G, 13H). After 6 months, the surgical site showed satisfactory healing and the temporary implants were well maintained without displacement (Fig. 14A). Subsequently, the secondary implant surgery was performed. After local anesthesia was administered, the temporary implants were removed using a wrench (Fig. 14B, 14C, 14D). Vertical and horizontal incisions were made in the middle and in both posterior regions and the flap was elevated to expose the implant cover screws (Fig. 14E, 14F). The cover screws were removed and the healing abutments were installed, followed by suturing (Fig. 14G). After soft tissue healing, the sutures were removed and a fixed implant-supported prosthesis was fabricated and installed (Fig. 14H). A panoramic radiograph obtained immediately after installation of the fixed implant-supported prosthesis showed good outcome (Fig. 15). A follow-up panoramic
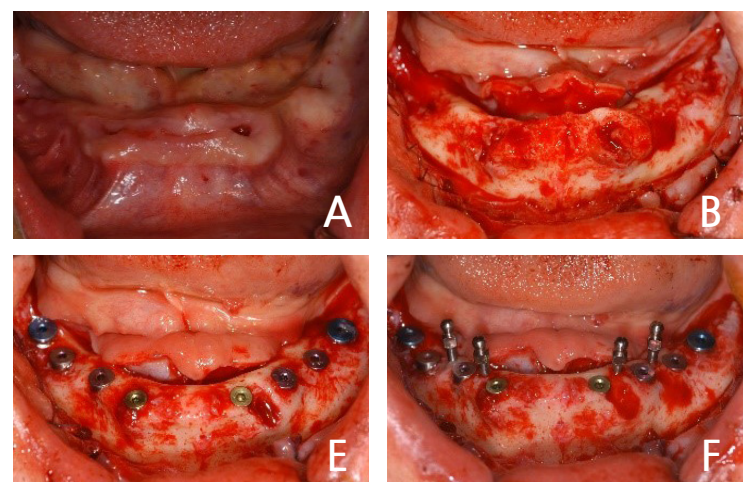
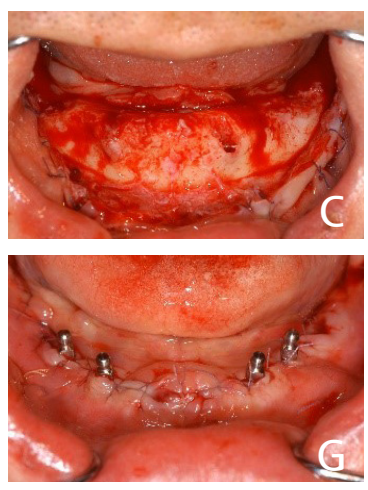

Fig. 13. Case II: Intraoral views of the first-stage implant surgery. (A) Pre-operative intraoral view, (B) full-thickness flap elevation, (C) smoothing of the uneven bone, (D) guide pin insertion, (E) implant placement, $(F)$ temporary implant insertion for temporary denture, $(G)$ suturing the flap, $(H)$ temporary denture delivery.
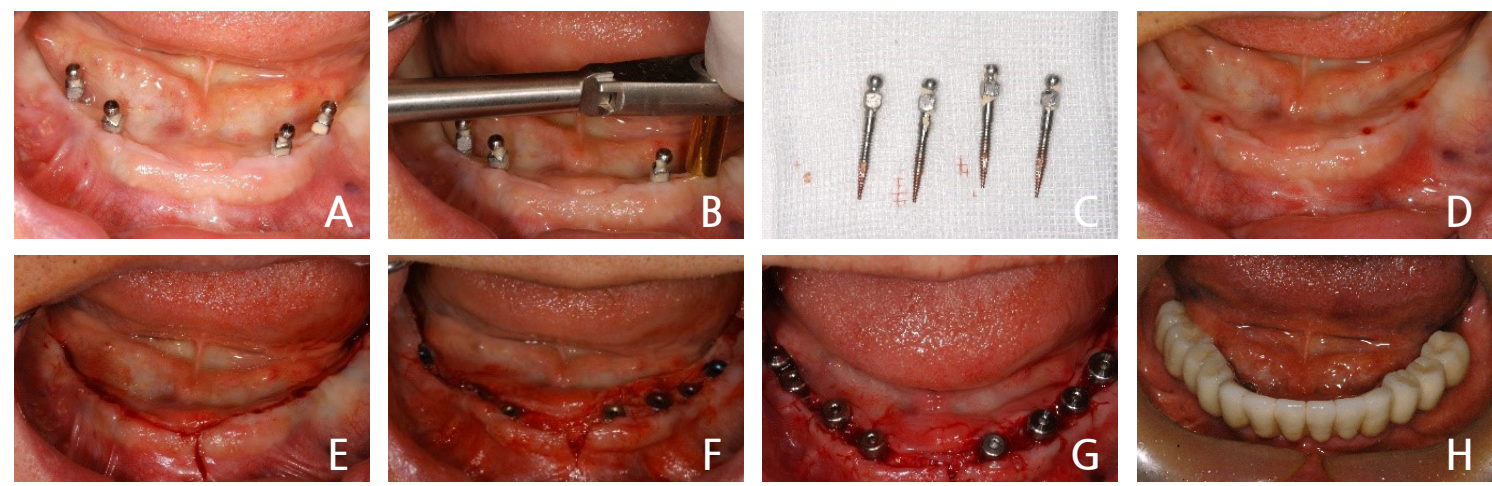

Fig. 14. Case II: Intraoral views of the second-stage implant surgery and the final restoration. (A) Preoperative intraoral view, (B) temporary implant removal, (C) temporary implant, (D) local anesthesia at the surgical site, $(E)$ incision at the surgical site, $(F)$ full-thickness flap elevation, $(G)$ healing abutment placement, $(\mathrm{H})$ final restoration. 


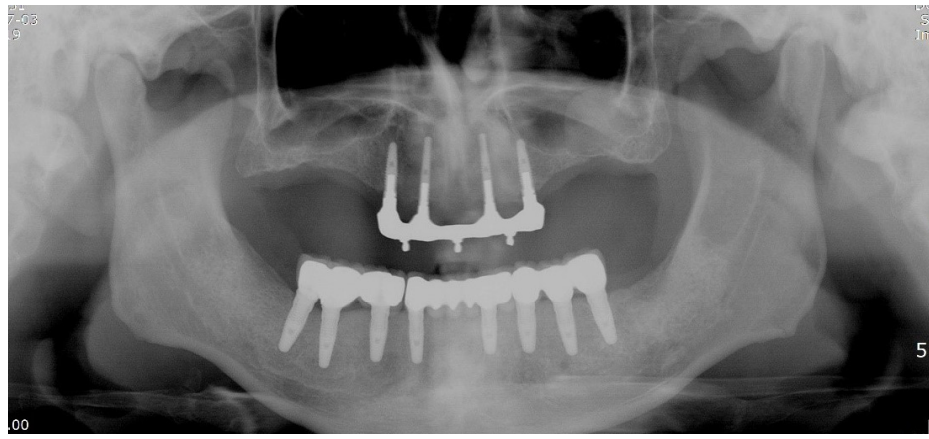

Fig. 15. Case Il: Panoramic radiograph of the final restoration.

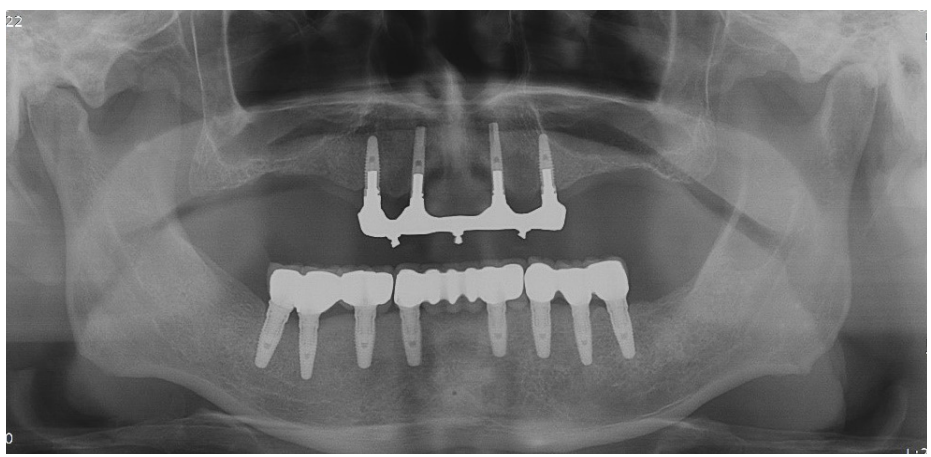

Fig. 16. Case Il: Panoramic radiograph of the final restoration after 8 years.

radiograph obtained after 8 years showed that the good outcome had persisted without marginal bone loss around the implants (Fig. 16).

\section{Discussion}

Dental implant restorations in edentulous patients aim to recover the lost hard and soft tissues in the oral cavity and damaged facial morphology. The decision to use a fixed implant-supported prosthesis or a removable prosthesis depends on determining the number of implants to be placed and the necessity and extent of bone grafts. Patients' needs, age, experience of wearing dentures, general health, economic status, and the condition of the opposing teeth need to be considered while establishing a treatment plan. ${ }^{7}$ Zitzmann and Marinello ${ }^{2}$ suggested that patients' preference, economic ability, pronunciation, and vomiting reflex should be considered as patient-associated factors while establishing a treatment plan. In the cases from the present report, patients preferred a fixed prosthesis. Restoration with a fixed prosthesis is advantageous in masticatory efficiency, patient satisfaction, and stress distribution to the remaining mandibular teeth when compared with a removable prosthesis. Hence, a fixed implantsupported prosthesis was preferred. 
Occlusal force applied to a prosthesis is affected by the condition of the opposing teeth. If the opposing teeth are natural teeth or a fixed restoration, the force applied to the prosthesis is greater. Therefore, a fixed prosthesis using multiple implants in the mandible and a complete denture in the maxilla may lead to accelerated progression of bone resorption in the maxilla due to the strong occlusal force generated by the mandibular prosthesis. In the present report, all maxillary opposing teeth were implant-supported removable overdentures. Treatment with a fixed prosthesis in the maxilla is very difficult when the upper lip support is insufficient or when the residual bone resorption is excessive. Restoration with an implantsupported removable overdenture can lead to better functional, esthetic, and hygienic outcomes.

If an edentulous jaw is restored with a fixed implant-supported prosthesis, it is necessary to consider in advance whether to recover the lost teeth and the surrounding tissues through a bone graft to a certain extent or to replace the lost area with a prosthetic restoration. The bone quality at the site of implant placement, the condition of the arch, and patients' economic ability should also be taken into consideration. Particularly, it is necessary to review various factors such as resorption pattern of the alveolar bone according to the period of denture use after extraction, the degree of adaptation to denture use, and the condition of the opposing teeth before treatment during treatment planning for completely edentulous mandible. Therefore, while planning an implant-supported restoration in a completely edentulous jaw, it is necessary to consider in advance whether to recover lost teeth and the surrounding tissues through a bone graft to a certain extent or to replace the lost area with prosthetic restoration. ${ }^{10}$ In the first case from this report, the patient was wearing a mandibular complete denture for a long time. Fortunately, the mandible had adequate length and thickness for implant placement. In the second case, tooth extraction was performed due to severe periodontitis. However, implants could be placed without bone graft, since the patient had an adequate amount of mandibular bone for implants.

Infection around an implant may occur after prosthetic restoration. It is broadly divided into periimplant mucositis, in which the lesion is localized to the soft tissue and peri-implantitis, in which the lesion extends to the bone tissue surrounding the implant. The former is a reversible inflammation showing soft tissue swelling, redness, and bleeding on probing, while the latter is an irreversible disease that can be accompanied by progressive bone resorption, periodontal pocket, edema, and abscess formation. ${ }^{11}$ In the first case of this report, peri-implantitis occurred around the implants in the left mandibular molar region at 10 years after the restoration and two implants needed to be removed. After the implants were replaced, a fixed implant prosthesis was fabricated, which has been well maintained for 5 years. In the second case, there have been no specific findings since the installation of the fixed implant-supported prosthesis and satisfactory functional and esthetic outcomes have been maintained. 


\section{IV . Conclusion}

In the clinical cases from this report, 12 and 8 implants were placed in the edentulous mandibles in case 1 and case 2 , respectively and fixed implant-supported prostheses were fabricated, resulting in good functional and esthetic outcomes over the 15-year and 8-year observation periods.

\section{References}

1. Lothigius E, Smedberg JI, De Buck V, Nilner K. A new design for a hybrid prosthesis supported by osseointegrated implants: 1. technical aspects. Int J Oral Maxillofac Implants 1991;6:80-6.

2. Zitzmann NU, Marinello CP. Treatment plan for restoring the edentulous maxilla with implantsupported restorations: removable overdenture versus fixed partial denture design. J Prosthet Dent 1999;82:188-96.

3. Thomason JM, Heydecke G, Feine JS, Ellis JS. How do patients perceive the benefit of reconstructive dentistry with regard to oral health-related quality of life and patient satisfaction? A systematic review. Clin Oral Implants Res 2007;18:168-88.

4. Kent G. Effects of osseointegrated implants on psychological and social well-being: a literature review. J Prosthet Dent 1992;68:515-8.

5. Brånemark PI, Svensson B, van Steenberghe D. Ten-year survival rates of fixed prostheses on four or six implants ad modum Branemark in full edentulism. Clin Oral Implants Res 1995;6:227-31.

6. Buser D, Ingimarsson S, Dula K, Lussi A, Hirt HP, Belser UC. Long-term stability of osseointegrated implants in augmented bone: a 5-year prospective study in partially edentulous patients. Int $\mathrm{J}$ Periodontics Restorative Dent 2002;22:109-17.

7. Wittneben JG, Avdic M, Wright RF, Radics A, Gallucci GO, Weber HP. Fixed mandibular and maxillary implant rehabilitation in a fully edentulous patient: a case report. Int J Periodontics Restorative Dent 2009;29:615-23.

8. Schroeder A, van der Zypen E, Stich H, Sutter F. The reactions of bone, connective tissue, and epithelium to endosteal implants with titaniumsprayed surfaces. J Maxillofac Surg 1981;9:15-25.

9. Taylor TD. Prosthodontic problems and limitations associated with osseointegration. J Prosthet Dent 1998;79:74-8.

10. Zarb GA, Schmitt A. The longitudinal clinical effectiveness of osseointegrated dental implants: the Toronto study. Part III: problems and complications encountered. J Prosthet Dent 1990;64:185-94.

11. Zitzmann NU, Berglundh T. Definition and prevalence of peri-implant diseases. J Clin Periodontol 2008;35:286-91. 